\title{
RECENT CHARACTERIZATIONS OF ELECTRON BEAMS FROM THE APS LINAC*
}

\author{
A.H. Lumpkin ${ }^{\dagger}$, W.J. Berg, M. Borland, and J. Lewellen \\ Advanced Photon Source, Argonne National Laboratory, Argonne, IL 60439 USA
}

\section{Abstract}

The Advanced Photon Source (APS) facility includes an S-band linear accelerator (linac) that normally provides beam for the injection system of the $7-\mathrm{GeV}$ storage ring. This beam is usually generated by one of two $\mathrm{rf}$ thermionic guns or the rf photocathode (PC) gun under special circumstances. When the beams are not required for injection, we may characterize them at a station located at the end of the linac. At this station we have begun a retrofit and upgrade of the optics on the diagnostics table that is outside of the linac enclosures. For beam transverse profiling studies, a CCD camera was used to image the beam spot at the YAG:Ce screen or the optical transition radiation (OTR) screen. In using the quadrupole field scanning technique we demonstrate that the YAG:Ce saturation effect is present under these conditions so that OTR imaging is needed for PC gun beam. A Hamamatsu C5680 streak camera with OTR and a zero-phasing rf technique with an electron spectrometer were used for bunch length measurements of beam from a newly-installed rf thermionic gun. These results will also be presented.

\section{INTRODUCTION}

Interest continues in the characterization of the linear accelerator (linac) beams at the Advanced Photon Source (APS) as components are upgraded or configurations changed. The APS facility includes an S-band linac that normally provides beam for the injection system of the 7$\mathrm{GeV}$ storage ring. This beam is generated by one of two rf thermionic guns or the rf photocathode (PC) gun under special circumstances. When the beams are not occupied with injection, we may characterize them at several stations along the accelerator. In particular, we report here observations at the end of the linac that were supported by a recent upgrade of the optics on the diagnostics table. The beam transverse profile measurements were performed at $325 \mathrm{MeV}$ using the PC gun beam and both YAG:Ce and optical transition radiation (OTR) screens. We demonstrated that during the quadrupole field scanning techniques used in the emittance measurements, the beam charge areal density crossed the saturation threshold of the YAG:Ce crystal so OTR imaging was needed [1]. We also evaluated the beam bunch length as a function of the scraper position in the alpha magnet associated with the rf thermionic gun. Both a streak camera technique with OTR and the zerophasing rf technique with an electron spectrometer were used. These measurements clearly showed that a shorter bunch length (albeit with less charge) could be obtained by scraping off the lower energy, later arriving electrons. A preliminary comparison of the results at the sub-ps level is presented.

\section{EXPERIMENTAL BACKGROUND}

These experiments were performed at the APS using beams accelerated by the S-band linac, which is normally used as part of the injector system for the $7-\mathrm{GeV}$ storage ring. The measurements were performed at the end of the linac at an energy of $325 \mathrm{MeV}$, the nominal energy for operations. As shown schematically in Fig. 1, either the rf thermionic gun or rf PC gun can be used to provide beam

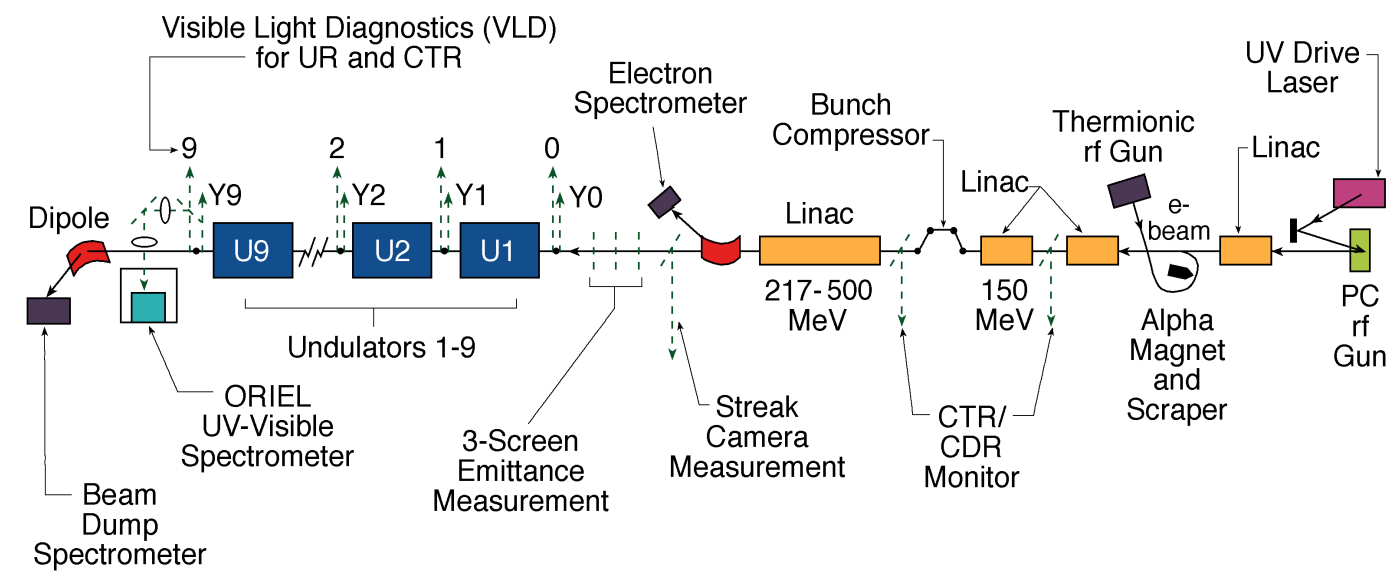

Figure 1: A schematic of the APS linac with its guns, accelerator, and diagnostic locations.

\footnotetext{
* Work supported by U.S. Department of Energy, Office of Basic Energy Sciences, under Contract No. W-31-109-ENG-38.

†lumpkin@aps.anl.gov
} 
to the linac. The thermionic gun system includes an alpha magnet for compression and injection of beam into the linac line. The PC gun beam can be compressed with a chicane bunch compressor as described elsewhere [2].

For the beam transverse profiling experiments, the beam was imaged at the same location indicated for the streak camera in Fig. 1 and just beyond the electron spectrometer. At the time of these experiments a crossed polarizer under EPICS control was added for remote light intensity control in front of the CCD camera that imaged the YAG:Ce screen or OTR screen. The visible light was transported by mirrors and a set of achromat lenses from the linac tunnel to the optics table outside of the radiation enclosure. In the case of the YAG:Ce screen tests, this remote intensity control was used during the quadrupole field scan to balance the video level signals generated. In the case of the OTR tests, the intensity was adjusted by the use of the neutral density filters to avoid the factor of 4 attenuation overhead of the crossed polarizers. The Vicon CCD was operated in the line-locked mode to provide a synchronization with the camera, line-locked linac, and the MaxVideo 200 video digitizer.

For the bunch length measurements, the Hamamatsu C5680 streak camera with OTR and the zero phasing rf technique with the electron spectrometer were used. The locations are shown in Fig. 1. Due to the low charge per micropulse $(\sim 30 \mathrm{pC})$ in the 8-ns-long macropulse from the rf thermionic gun, the streak camera images were taken in synchroscan mode at $119.0 \mathrm{MHz}$ as a 10-shot or micropulse average. However, the Chromox converter screen in the focal plane of the electron spectrometer has a slow time response that averaged the effects of all 25 micropulses in each macropulse. By adjusting the $\mathrm{rf}$ accelerator phase in a section upstream of the spectrometer so the micropulse arrived on the maximum slope of the rf cycle, the arrival times of the electrons were encoded as energy differences. The energy plane became a time axis for the micropulses. Our initial experiments with the newly installed gun were to evaluate the effects of the alpha-magnet scraper position on observed micropulse bunch length and to compare the two measurement techniques.

\section{EXPERIMENTAL RESULTS}

In this section we will present the results of the transverse and longitudinal beam profiling experiments.

\section{Transverse Beam Profile Results}

One of the goals of these beam profiling experiments was to determine if the YAG:Ce converter screen could be reliably used for beam characterizations of the PC gun beam at this location. We rely on beam size measurements based on YAG:Ce screen at $150 \mathrm{MeV}$ after the chicane to evaluate emittance and in the PAR bypass regime. We had previously discovered an effective threshold for saturation, where above certain areal charge densities the screen gives a blurred image [1]. The quadrupole scans were first done with the YAG:Ce screen and then repeated with the OTR screen. The image intensities were adjusted so that we operated with similar video levels into the video digitizer. The results of the scans are shown in Fig. 2. The YAG:Ce images are systematically a little larger than the OTR images, and this is particularly true at the beam waist. The saturation effect results in an overestimate of the PC gun beam size by a factor of 1.75 . This is again a demonstration that tightly focused beams of $300 \mathrm{pC}$ per pulse can exceed the saturation threshold in YAG:Ce crystals, so care must be taken in employing such screens for beam characterizations.

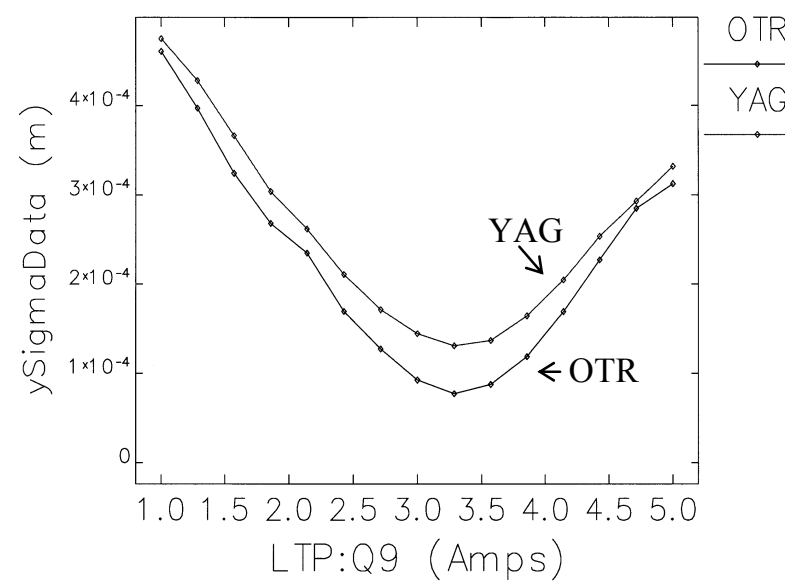

Figure 2: A comparison of beam sizes measured as a function of quadrupole field strength using the YAG:Ce screen and the OTR screen. Smaller beam sizes are observed with the OTR technique at the beam waist.

\section{Bunch Length Measurement Results}

Our newly-installed rf thermionic gun (\#2) was next evaluated. The macropulse contained about $0.7 \mathrm{nC}$ with the scraper at the out position (reading $9.5 \mathrm{~cm}$ ). At this position an rms bunch length of about 2 ps was measured by both techniques. An example of the streak image is given in Fig. 3, and a tabulation of results for several images and conditions is provided in Table 1. As the scraper is put further into the beam in the dispersive point of the alpha magnet, the low-energy tail and then the core on the low energy/later time side are trimmed. At the $11.5-\mathrm{cm}$ scraper position a very narrow peak at about the expected streak camera resolution limit of $0.6 \mathrm{ps}$ is observed. There is some evidence of the gun or $\mathrm{rf}$ transitioning between conditions that result in two bunch length states. A bandpass filter with $40-\mathrm{nm}$ width was used to reduce the chromatic temporal effects in the streak camera input optics.

Statistical fluctuations seemed reduced in the zerophasing rf technique data. Evaluations of the bunch length vs. scraper position are shown in Fig. 4. The shortest bunch length calculated at the scraper setting of $11.7 \mathrm{~cm}$ is below the streak camera resolution. However, we believe these initial cross comparisons of the two techniques also contributed to the gun characterization. 
Table 1: A Tabulation of Preliminary Streak Camera Results versus Alpha-Magnet Scraper Settings

\begin{tabular}{|c|c|c|c|c|c|}
\hline Run \# & $\begin{array}{c}\text { Scraper } \\
\text { Position } \\
(\mathbf{c m})\end{array}$ & $\begin{array}{c}\text { Charge: } \\
\text { L5 cm1 } \\
(\mathbf{n C})\end{array}$ & $\begin{array}{c}\text { Raw FWHM } \\
\mathbf{( p s )}\end{array}$ & \# Averaged & $\begin{array}{c}\text { Raw } \\
\text { Estimated } \boldsymbol{\sigma} \\
(\mathbf{p s})\end{array}$ \\
\hline $\mathrm{A}$ & 9.5 & 0.7 & 4.6 & 1 & 2.0 \\
\hline $\mathrm{A}$ & 11.0 & 0.6 & 3.0 & 2 & 1.3 \\
\hline $\mathrm{A}$ & 11.3 & 0.4 & 2.6 & 2 & 1.1 \\
\hline $\mathrm{B}$ & 9.5 & 0.8 & 5.1 & 6 & 2.2 \\
\hline $\mathrm{B}$ & 11.0 & 0.7 & 4.1 & 2 & 1.7 \\
\hline $\mathrm{B}$ & 11.5 & 0.3 & 3.4 & 2 & 1.5 \\
& & & 2.4 & 3 & 1.0 \\
\hline
\end{tabular}

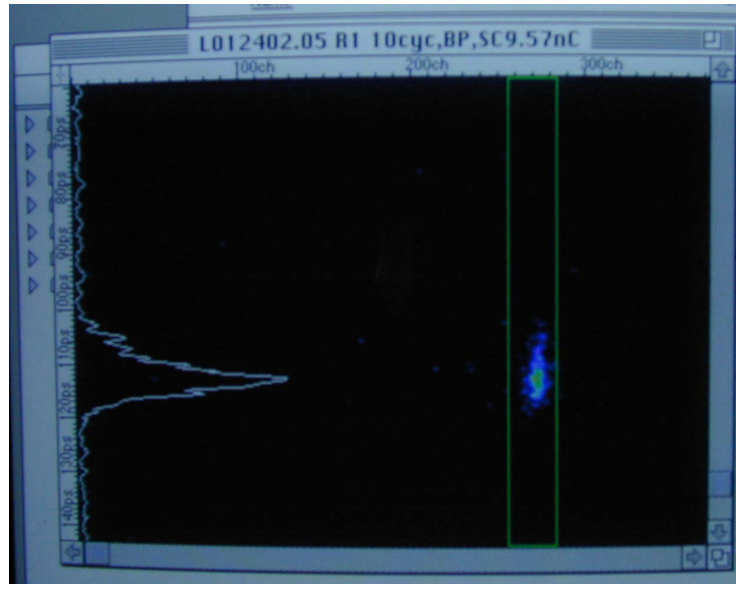

Figure 3: An example of a streak image of the $\mathrm{rf}$ thermionic gun beam for a scraper setting of $9.5 \mathrm{~cm}$. The time axis is the vertical axis, and the x-profile is on the horizontal axis.

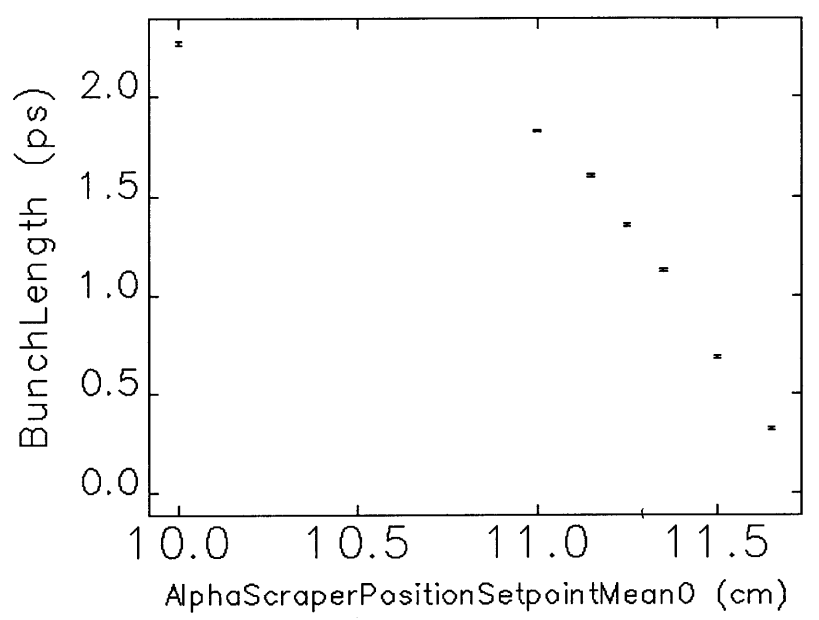

Figure 4: A plot of the measured bunch length using the zero-phasing rf technique vs. the alpha-magnet scraper position.

\section{SUMMARY}

In summary, we have used our imaging techniques at the end-of-linac station (sta-5) to determine the limitation of the YAG:Ce screen for small, bright beams at 325 $\mathrm{MeV}$. In addition, we have performed the first bunch length measurements as a function of alpha-magnet scraper position for our new rf thermionic gun. Sub-ps bunch lengths were obtained, although at very low charge per micropulse.

\section{ACKNOWLEDGMENTS}

The authors acknowledge the support of O. Singh, G. Decker, and R. Klaffky of the Accelerator Operations Division of APS

\section{REFERENCES}

[1] A.H. Lumpkin et al., Nucl. Instrum. Methods in Phys. Res. A429, 336-340 (1999).

[2] M. Borland, J. W. Lewellen, and S.V. Milton, "A Highly Flexible Bunch Compressor for the APS LEUTL FEL," Proc. of the XX International Linear Accelerator Conference (LINAC2000), SLAC-R-561, 863-865 (2001). 